\title{
What Information Theory Can Tell Us About Quantum Reality
}

\author{
C. Adami and N.J. Cerf \\ W. K. Kellogg Radiation Laboratory \\ California Institute of Technology, Pasadena, California 91125, USA
}

\begin{abstract}
An investigation of Einstein's "physical" reality and the concept of quantum reality in terms of information theory suggests a solution to quantum paradoxes such as the Einstein-Podolsky-Rosen (EPR) and the Schrödinger-cat paradoxes. Quantum reality, the picture based on unitarily evolving wavefunctions, is complete, but appears incomplete from the observer's point of view for fundamental reasons arising from the quantum information theory of measurement. Physical reality, the picture based on classically accessible observables is, in the worst case of EPR experiments, unrelated to the quantum reality it purports to reflect. Thus, quantum information theory implies that only correlations, not the correlata, are physically accessible: the mantra of the Ithaca interpretation of quantum mechanics.
\end{abstract}

\section{Introduction}

The concept of "physical reality" as championed by Einstein [1 - the postulate that the objective state of a system is specified by a set of real-valued parameters independently of our knowledge of them - has been an object of contention ever since the inception of quantum theory (see, e.g., [2, 3, 4, 5, 6, 6, ,7). The most prevailing views assert either that the "quantum reality" suggested by wavefunctions and non-local correlations is only a mathematical construction necessary for a consistent theory (Bohr's view), or else that physical reality is deterministic but incompletely described by quantum mechanics (Einstein's view). A popular interpretation of the latter view is that physical reality is obscured by inaccessible hidden variables [8], a stance that appears to be discredited by the violation of Bell's inequalities in quantum mechanics [9]. Bohr's view of complementarity, on the other hand, assigns a special status to classical physics as an essential ingredient in measurement since it requires the measurement device to be classical. As recognized by von Neumann [10], this undermines the foundations of quantum mechanics as a complete and consistent theory. Here, we suggest that Einstein realism and Bohr's complementarity principle can be reconciled within a framework that consistently describes the concept of information in quantum mechanics. This is exemplified by the quantum information theoretic treatment of the Einstein-Podolsky-Rosen (EPR) experiment [1] and the Schrödinger-cat paradox [11], which has recently attracted increasing attention (see, e.g., [12]). We propose that, in general, the perceived physical reality and quantum reality can 
be disjoint, that is, the result of a quantum measurement conceivably might not carry any information - in the sense of Shannon theory $[13$ - which would allow the observer to infer the state of the measured system. While counterintuitive, we shall show that this picture is a direct consequence of an information-theoretic reinterpretation of quantum measurement. Moreover, such a view effortlessly resolves the EPR paradox which has inspired the discussions on reality, as well as other quantum paradoxes rooted in the measurement problem.

The gedankenexperiment that constitutes the EPR paradox was created by Einstein, Podolsky, and Rosen to demonstrate their dissatisfaction with "unknowables" [1]. In that experiment, it appears that two complementary variables (such as position and momentum) are in principle measurable by exploiting the quantum correlations between the two particles, in contradiction with Heisenberg's uncertainty principle. Their conclusion, namely that the quantum mechanical description of reality must therefore be incomplete, was based on a criterion for reality which they considered "reasonable" (see below). This criterion was faulted by Bohr 14 in his reply to the EPR paper, insisting rather that physical variables are never independent of the way they are measured owing to the complementarity principle, and therefore that measurements do not confer reality to properties of quantum objects. We shall show here, using quantum information theory only, that, while indeed an element of reality is not created for the measured quantum system, the result of a quantum measurement creates an element of reality for the result of another measurement, i.e., it allows you to predict the state of another measurement device without revealing the state of the quantum system itself. Thus, physical reality is reflected in correlations between classical objects only. This view, which we arrived at from a quantum information-theoretic examination of quantum measurement 15.,16] essentially coincides with Mermin's "Ithaca Interpretation of Quantum Mechanics", Ref. [17].

\section{The EPR Paradox}

The EPR experiment in the version of Bohm [18 involves the preparation of a quantum system such as the one created by the decay of a spinless particle into two half-integral-spin particles:

$$
\left|\Psi_{\mathrm{EPR}}\right\rangle=\frac{1}{\sqrt{2}}(|\uparrow \downarrow\rangle-|\downarrow \uparrow\rangle) .
$$

This state represents the superposition of the two possible situations: "leftparticle spin-up, right-particle spin-down", and "right-particle spin-up, left-particle spin-down". Let us now imagine that the pair so-created is separated sufficiently far that classical information would take a long time to travel between them. Then, we measure for example the $z$-component of the spin of one of the particles (say, the left one). This measurement has two possible outcomes, which occur with probability one-half each, implying that the von Neumann uncertainty of the density matrix describing any one of the particles (denoted by 
subscripts $L$ and $R$ ),

$$
\rho_{L, R}=\frac{1}{2}|\uparrow\rangle\left\langle\uparrow\left|+\frac{1}{2}\right| \downarrow\right\rangle\langle\downarrow|
$$

is one bit

$$
S\left(\rho_{L, R}\right)=-\operatorname{Tr}_{\mathrm{R}, \mathrm{L}}\left(\rho_{L, R} \log _{2} \rho_{L, R}\right)=1
$$

in spite of the fact that entropy of the combined system vanishes. The latter is of course well-known: for a quantum mechanical "pure state" $\left(\rho_{\mathrm{EPR}}^{2}=\rho_{\mathrm{EPR}}\right.$, where $\left.\rho_{\mathrm{EPR}}=\left|\Psi_{\mathrm{EPR}}\right\rangle\left\langle\Psi_{\mathrm{EPR}}\right|\right)$ the von-Neumann entropy vanishes $S\left(\rho_{\mathrm{EPR}}\right)=0$, i.e., the state is perfectly well-known.

Clearly then, the quantum nature of the EPR state is very peculiar since the uncertainty of a part of this system can be larger than the uncertainty of the pair. Classically, this is impossible. Indeed, if we describe uncertainties using (classical) Shannon entropies, the Shannon entropy of a system $A$, say, with $A \subset A B$, is

$$
H(A) \leq H(A B) .
$$

This property of monotonicity of entropies is violated in quantum mechanics [19]. This violation, on the other hand, can be described consistently in an information-theoretic formalism which allows for negative conditional entropies 20,21]. In other words, there exists an information theory, extended to the quantum regime, in which the violation of classical laws such as monotonicity are inevitable consequences.

Quantum entanglement situations, such as encountered in EPR pairs, are prototype systems to examine the classically forbidden regime of negative entropies. In the case at hand, the joint, conditional, mutual, and marginal entropies of the EPR pair can be summarized by the entropy diagram in Fig. 1. Such diagrams are used extensively in classical information theory and serve as mental scratch pads to remind us of the separation of unconditional entropies into conditional and mutual pieces. While in the past the violation of monotonicity prevented the use of Venn diagrams in quantum information theory, the introduction of negative entropies has reinstated this useful tool [20,21, 15, 16]. In particular, we can see how

$$
S(L) \not \leq S(L R) \text {. }
$$

is possible in Fig. 1 if $S(L \mid R)$ is negative.

The repercussions of such an information-theoretic description of entanglement for the extraction of information from such a state (a measurement) are manifold. Here, we focus on EPR experiments and other quantum paradoxes, and on implications for physical as well as quantum pictures of reality.

\section{Information Theory of EPR Experiments}

In order to assess the relation between quantum and physical reality in an EPR measurement, we need to describe both the quantum system (the EPR wavefunction) and the classical devices it becomes entangled with, using information theory. 
Fig. 1. Quantum entropy diagrams. (a) Definition of joint $[S(L R)]$ (the total area), marginal $[S(L)$ or $S(R)]$, conditional $[S(L \mid R)$ or $S(R \mid L)]$ and mutual $[S(L: R)]$ entropies for a quantum system $L R$ separated into two subsystems $L$ and $R$; (b) their respective values for the EPR pair.

(a)

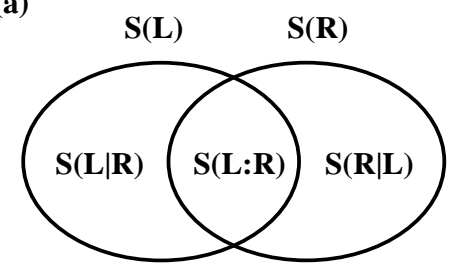

(b)

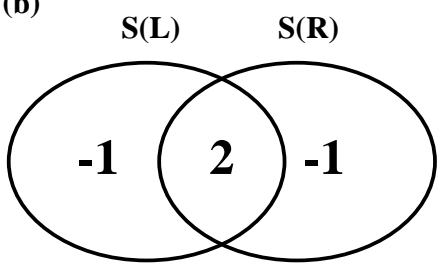

Let $A_{1}$ and $A_{2}$ denote measurement devices, each of the devices measuring the $z$ component of one member of an EPR pair, for example (see Fig. 2).

Fig. 2. Measurement of EPR pair $Q_{1} Q_{2}$ by devices $A_{1}$ and $A_{2}$.
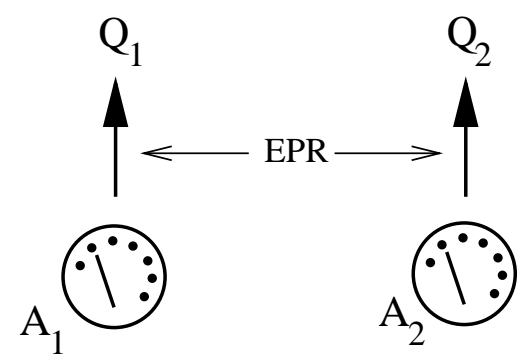

It is an experimental fact that the measurement of the state of one of the particles (say, $\sigma_{z}$ ) allows a $100 \%$ accurate prediction of what the outcome of the measurement of the other one will be. Thus, the outcomes of the measurement of $\sigma_{z}$ are perfectly correlated, a situation described by the entropy diagram in Fig. 3a, which appears perfectly classical (no negative numbers appear).

Note that the correlations between the devices are quite unlike those of the quantum system that is measured, a peculiarity that is quantitatively manifested when comparing Figs. 1b and 3a. The reason why the correlations between the measurement devices (Fig. Ba) incompletely mirror the entanglement present in the quantum state (Fig. 1b) must be due to the device's classical nature: classical conditional entropies cannot be negative. However, classicality must not be assumed for the devices, it is a mathematical result of the informationtheoretic treatment of measurement (which gives rise to Fig. 3a) [16].

Assume now that orthogonal spin projections are measured on the two halves of the EPR pair, say $\sigma_{z}$ on the left particle, and $\sigma_{x}$ on the right one. If we assume that measuring the state of one partner confers reality to the state of the 
measured system, we must conclude that the experiment just described would allow us to infer the $z$ and $x$ projections simultaneously, a state of affairs strictly

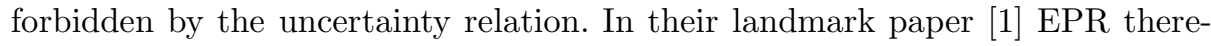
fore conclude that, since conventional quantum mechanics cannot describe this peculiar situation, the theory must necessarily be incomplete. This is the essence of the EPR paradox. It relies on a definition of reality based on "certain prediction" " according to which the state of the second particle would acquire physical reality after measuring its EPR partner. In fact, for this particular experiment (measuring $\sigma_{z}$ on the left and $\sigma_{x}$ on the right particle) it is found that the outcomes recorded by the devices are completely uncorrelated as depicted by the classical entropy diagram for the devices pictured in Fig. 3b. Rather than reflecting an incompleteness of the formalism, these outcomes are predicted by quantum information theory, and imply that physical reality is attributed to the state of the second measurement device, or more precisely the relative state of the devices, while there cannot be any correlation between the apparatus and the quantum state proper (as we show below). In view of the importance of this conclusion, let us repeat it once more. Quantum information theory predicts that in EPR-type measurements, the measurement device cannot reflect the state of of the quantum system. In the language of Mermin [17, the correlations between the devices are real, i.e., possess physical reality, while the quantum system itself does not.

Fig. 3. Entropy diagram for the devices: (a) recording $\sigma_{z}$ for each of the entangled particles, or (b) recording $\sigma_{z}$ for one and $\sigma_{x}$ for the other particle.

(a)

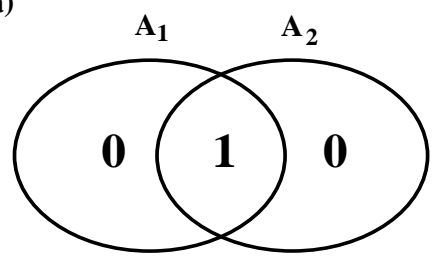

(b)

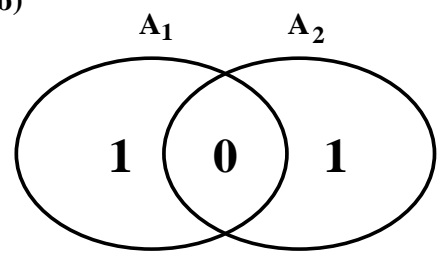

Let us show this in more detail. For a proper quantum information-theoretic analysis, we need to consider four systems: a quantum pair $Q_{1} Q_{2}$ and a pair of ancillae $A_{1} A_{2}$. The ancillae can be thought of as classical devices that are built to reflect the quantum states. From a measurement point of view, we are interested in the correlations between the ancillae, as only such correlations are experimentally accessible (relative states). Before we analyze them using quantum entropy diagrams, let us ponder what we expect to find from an orthodox point of view.

\footnotetext{
${ }^{1}$ EPR wrote in 11]: "If, without in any way disturbing a system, we can predict with certainty (i.e., with probability equal to unity) the value of a physical quantity, then there exists an element of physical reality corresponding to this physical quantity."
} 
One of the fundamental tenets of classical measurement theory is that a measurement device is constructed such as to mirror the state of the object to be measured as accurately as possible. In other words, measurement entails the transfer of this information to a macroscopic system that is more suited to accurate observation, without altering the state of the system. While it is wellknown that quantum measurements cannot be made without altering the quantum state 22, the general belief is that the quantum state after measurement $i s$ truthfully portrayed by the device. In other words, it is believed that correlations between the quantum state and the ancillae in the measurement situation allow the extraction of information about the quantum system. Let us consider the "orthodox" (classical) entropy diagram (Fig. 14) for an EPR measurement, drawing the quantum system $Q_{1} Q_{2}$ as one system, measured by the ancillae $A_{1}$ and $A_{2}$. These diagrams reveal the paradox inherent in this description. On the

Fig. 4. Classical entropy diagram for the EPR measurement of spin-projections: (a) both devices measure $\sigma_{z}$, (b) one device measures $\sigma_{z}$, the other $\sigma_{x}$. Note that the entropy of $A_{1}$ and $A_{2}$ have to be one bit in each case, as the measurement outcomes are equiprobable, while $Q_{1} Q_{2}$ is thought to have two independent equiprobable degrees of freedom.

(a)

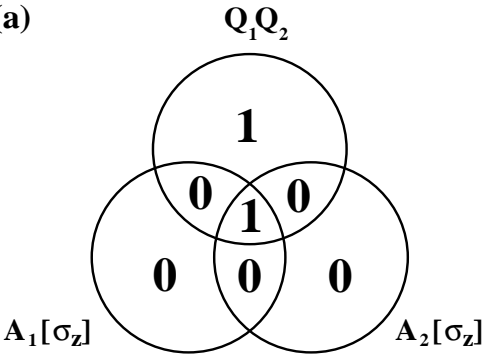

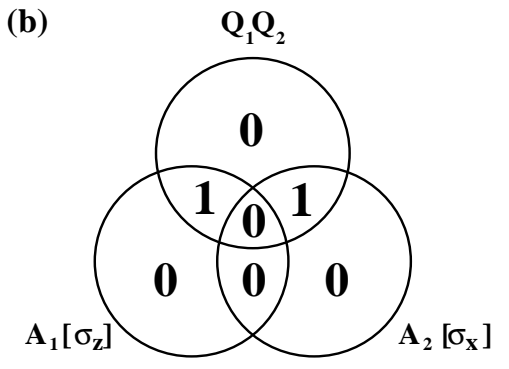

one hand, when the same projection of the spin (e.g., $\sigma_{z}$ ) is measured for both particles (Fig. Aa) classical reasoning suggests that the quantum system and the measurement devices share information (one bit in the center of the diagram). On the other hand, when orthogonal polarizations are measured (Fig. 4 $\mathrm{b}$ ) the measurement devices must appear uncorrelated. According to a "physical realism" or "hidden variable" picture, both diagrams in Fig. A must have a common underlying classical diagram relating five ensembles: the EPR pair $Q_{1} Q_{2}$ and the four possible measurements $A_{1}\left[\sigma_{z}\right], A_{1}\left[\sigma_{x}\right], A_{2}\left[\sigma_{z}\right]$, and $A_{2}\left[\sigma_{x}\right]^{2}$. This underlying diagram, however, is in contradiction with the Heisenberg uncertainty principle, as it implies that the counterfactual variables $\sigma_{x}$ and $\sigma_{z}$ (of the same particle)

\footnotetext{
${ }^{2}$ The diagrams in Fig. 1 are obtained from such an underlying diagram by ignoring two out of the five variables: Fig. $4 \mathrm{a}$ by ignoring $A_{1}\left[\sigma_{x}\right]$ and $A_{2}\left[\sigma_{x}\right]$, Fig. $4 \mathrm{~b}$ by ignoring $A_{1}\left[\sigma_{x}\right]$ and $A_{2}\left[\sigma_{z}\right]$. "Ignoring" a system is achieved by the mathematical operation of tracing it out of the joint density matrix.
} 
Fig. 5. (a) Quantum entropy diagram for the EPR measurement of same spinprojections: e.g., $A_{1}$ and $A_{2}$ both measure $\sigma_{z}$. (b) reduced diagram obtained by tracing over the quantum states $Q_{1}$ and $Q_{2}$.
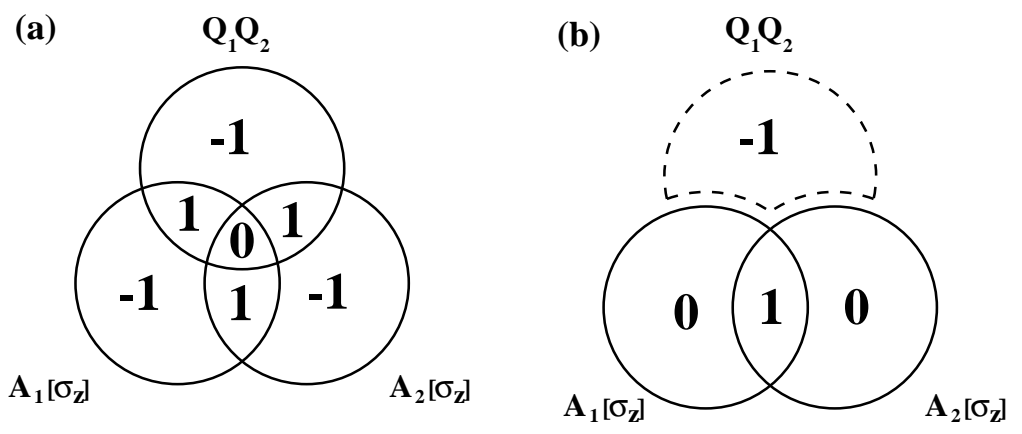

can be measured together. Thus, this classical treatment of information leads to a paradox.

The paradox is resolved by drawing the quantum entropy diagrams for the measurements (Figs. 5 and 6). The values for the respective quantum entropies entering these diagrams can be obtained by straightforward calculation [16]. In Fig. 5 the entropy diagram representing the situation where the same polarizations are measured is that of a GHZ state [23]: fully symmetric and maximum quantum entanglement between three entities. As is well-known, tracing over (ignoring) one member of such a triplet produces classical correlations (of the type depicted in Fig. 3a) in the remaining doublet, as indicated in Fig. 5b. As a consequence, the quantum entropy diagram of Fig. Fa correctly reproduces the observed correlations between the detectors $A_{1}$ and $A_{2}$. Closer inspection of Fig. Fa, however, reveals that while the measurement devices are perfectly correlated as they should, their mutual entropy (the single bit of information gained in the measurement) is not shared by the quantum system $Q_{1} Q_{2}$. In Figs. . and 6. this ternary mutual information ${ }^{[}$is represented by the center of the diagram, and measures how much of the correlations between the measurement devices is shared by the quantum system. If the center of the diagram is zero, we must conclude that no information is shared between quantum system and classical devices.

The same is true for the measurement situation in Fig. 6a, where incompatible polarizations are measured. Again, the (four part) system is fully entangled, and ignoring the quantum state produces the experimentally observed independence of the measurement results (Fig. $6 \mathrm{~b}$, compare Fig. $3 \mathrm{~b}$ ). Yet, the correlation between quantum state and measurement device (the mutual information between

\footnotetext{
${ }^{3}$ Just like any entropy, information, which is the mutual entropy between two systems, can be split up into a conditional and a mutual piece with respect to a third system 13.
} 
the measuring and the measured system) is unchanged from the previous arrangement, in fact, it vanishes in both cases 1 .

Fig. 6. (a) Quantum entropy diagram for the EPR measurement of orthogonal spin-projections, e.g., $A_{1}$ measures $\sigma_{x}$ while $A_{2}$ records $\sigma_{z}$. (b) Reduced diagram as above.
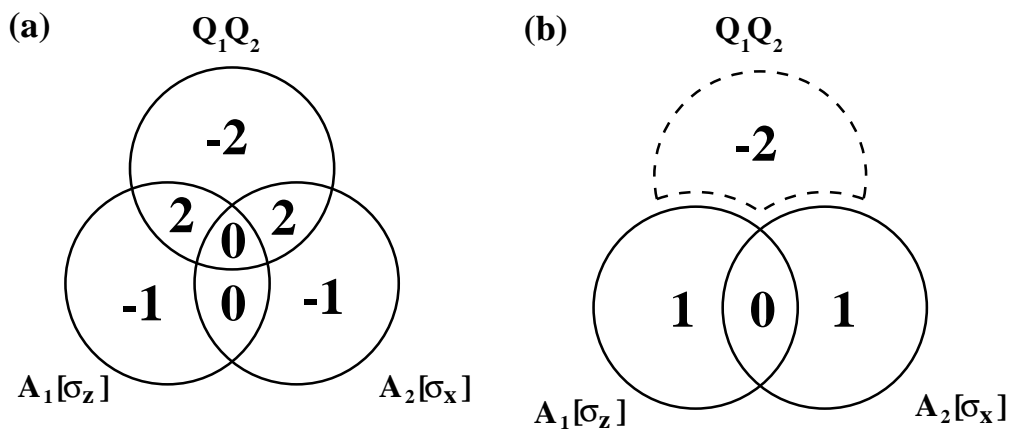

This situation leads us to suggest that we must abandon at least one cherished notion of orthodox measurement theory: that the apparatus necessarily reflects the state of the system it was built to measure, by being correlated with it in the sense of Shannon. Rather, it is the correlations between the ancillae (the reality of their relative state) that create the illusion of measurement. Indeed, any subsequent measurement on each side (left or right), for example, would yield the same result, over and over again, while still not implying anything about the quantum wavefunction. Each observer that repeats a measurement becomes classically correlated to the earlier outcome, whatever the outcome. Still, the quantum reality of the superposition is unperturbed by these measurements: none of the repeatable measurements yield any information about the quantum state, while they are internally completely consistent. Note that the orthodox interpretation of these correlations involves the concept of a wavefunction collapse: the measurement of the first particle projects - or collapses - the wavefunction of the other one, to account for the perfect correlation. Since the devices do not reflect the state of the quantum system, however, no collapse is needed to explain the correlations, nor does it actually occur, as we now show.

\section{Information Theory of Schrödinger Cats}

The Schrödinger-cat paradox is of prime importance for the understanding of quantum decoherence and the quantum-classical boundary. The latter have received increased attention recently due to their importance for the design of quantum computation and communication devices [24].

\footnotetext{
${ }^{4}$ The mutual information between quantum system and both classical devices also vanishes for any intermediate situation between Figs. 5 and 6 , since the joint system $Q_{1} Q_{2} A_{1} A_{2}$ is always a pure state [16].
} 
The Schrödinger-cat paradox explores the relationship between classical and quantum variables by coupling them together in such a way that the decay of a radioactive substance (say, one isolated atom) implies the demise of a cat locked up with the deadly contraption in a sealed room. The quantum reality of the (isolated) atomic system is that of a superposition of a decayed atom with gamma ray, and an undecayed atom without. If brought into contact with the cat, however, quantum mechanics forces us to include the cat in this entangled wavefunction

$$
|\Psi\rangle=\frac{1}{\sqrt{2}}\left(\left|A^{\star}, 0, L\right\rangle+|A, 1, D\rangle\right),
$$

where $\left|A^{\star}\right\rangle$ and $|0\rangle$ refer to the excited atom and absent gamma, while $|A\rangle$ and $|1\rangle$ are the wavefunctions of the decayed atom and the gamma. Furthermore, $|L\rangle$ and $|D\rangle$ refer to the "live" and "dead" cat eigenstates. The paradox arises if an observer peeks into the room to observe the state of the cat. Does the cat's wavefunction immediately collapse into one of its eigenstates (dead or alive) upon observation? The preceding analysis teaches us that this is not necessary. The observer can be thought of as a fourth system that is now quantum entangled with the previous troika: atom, gamma, and cat

$$
|\Psi\rangle=\frac{1}{\sqrt{2}}\left(\left|A^{\star}, 0, L, l\right\rangle+|A, 1, D, d\rangle\right),
$$

introducing "observer eigenstates" $|l\rangle$ and $|d\rangle$. Then, upon tracing over the quantum degrees of freedom of the atom (after all, this experiment involves monitoring the cat and not the atom), the cat (serving as the hapless gamma-ray detector) appears perfectly correlated with the observer peeking into the room. Cat and observer agree, so to speak, about the observation, and their state is entirely classical. Yet, their agreement is completely decorrelated, disjoint, from the quantum state, as their mutual information shared with the atomic system vanishes. In other words, the classical reality displayed by cat and observer does not imply anything about the quantum reality of atom and gamma ray, or vice versa. Fundamentally, the reason why the observer does not register a cat mired in a quantum superposition of the living and non-living states is because the observer, having interacted with the cat, is entangled with, and thus part of, the same wavefunction. As the wavefunction is indivisible, an observer (or measurement device) would have to monitor itself in order to learn about the wavefunction. This is logically impossible.

\section{Conclusions}

To summarize, we assert that quantum reality is "real" in the sense that quantum mechanics completely and deterministically describes the evolution of a closed system (not just its wavefunction), and that the statistical character arises from the fact that an observer, because he is part of the closed system, is offered an

incomplete view of the quantum system he attempts to measure. Consequently, the quantum universe is deterministic as Einstein's physical reality demands, 
but must include the observer as one of its parts due to the inseparability of entangled quantum states. The recent information-theoretic analysis of quantum measurement [15,16] shows that such an observer indeed perceives the system he is measuring as probabilistic, and thus that Bohr's complementarity principle emphasizing the importance of the system/observer relation therefore holds at the same time. If quantum reality is so elusive, how then can we learn about its nature? Fortunately, while negative entropy cannot be reflected in classical instruments directly, it is possible to infer it from combined measurements and comparison with classical bounds (a case in point are Bell inequalities [9], see also 25]). Thus, quantum reality does leave its traces in experiments, while the direct observation of superpositions is impossible.

\section{Acknowledgments}

This work was supported in part by NSF Grant Nos. PHY 94-12818 and PHY 9420470 and by a grant from DARPA/ARO through the QUIC program (\#DAAH0496-1-3086). N.J.C. is Collaborateur Scientifique of the Belgian National Fund for Scientific Research. An earlier version of this paper was circulated in the Fall of 1996 under the title "Physical Reality and Quantum Paradoxes".

\section{References}

1. A. Einstein, B. Podolsky, and N. Rosen, Can quantum-mechanical description of physical reality be considered complete? Phys. Rev. 47, 777 (1935).

2. N. Bohr, The quantum postulate and the recent development of atomic theory, Nature 121, 580 (1928).

3. J.A. Wheeler and W.H. Zurek, eds., Quantum Theory and Measurement (Princeton University Press, 1983).

4. J.S. Bell, Speakable and Unspeakable in Quantum Mechanics (Cambridge University Press, Cambridge, 1987).

5. W. Schommers, ed., Quantum Theory and Pictures of Reality, (Springer, Berlin, 1989).

6. P. Busch, P.J. Lahti, and P. Mittelstädt, The Quantum Theory of Measurement (Springer, New York, 1991).

7. J.T. Cushing, Quantum Mechanics-Historical Contingencies and the Copenhagen Hegemony, (University of Chicago Press, Chicago, 1994).

8. D. Bohm, A suggested interpretation of the quantum theory in terms of hidden variables, I and II, Phys. Rev. 85, 166 (1952).

9. J.S. Bell, On the Einstein Podolsky Rosen paradox, Physics 1, 195 (1965).

10. J. von Neumann, Mathematische Grundlagen der Quantenmechanik (Springer Verlag, Berlin, 1932).

11. E. Schrödinger, Die gegenwärtige Situation in der Quantenmechanik, Naturwissenschaften 23, 807 (1935).

12. C. Monroe, D. M. Meekhof, B.E. King, and D. J. Wineland, A Schrödinger cat superposition state of an atom, Science 272, 1131 (1996); J. J. Slosser and G.J. Milburn, Creating metastable Schrödinger cat states, Phys. Rev. Lett. 75, 418 (1995). 
13. C.E. Shannon and W. Weaver, The Mathematical Theory of Communication (University of Illinois Press, 1949).

14. N. Bohr, Can quantum-mechanical description of physical reality be considered complete?, Phys. Rev. 48, 696 (1935).

15. N.J. Cerf and C. Adami, Quantum mechanics of measurement, eprint quant$\mathrm{ph} / 9605002$, unpublished.

16. N.J. Cerf and C. Adami, Information theory of quantum entanglement and measurement, Physica D (1998).

17. N.D. Mermin, What is quantum mechanics trying to tell us?, eprint quant$\mathrm{ph} / 9801057$.

18. D. Bohm, Quantum Theory, (Prentice-Hall, Englewood Cliffs, 1951), pp. 611-623.

19. A. Wehrl, General properties of entropy, Rev. Mod. Phys. 50, 221 (1978).

20. N.J. Cerf and C. Adami, Negative entropy and information in quantum mechanics, Phys. Rev. Lett. 79 (1997) 5194.

21. N.J. Cerf and C. Adami, Negative entropy in quantum information theory, in New Developments on Fundamental Problems in Quantum Physics, Fundamental Theories of Physics 81, M. Ferrero and A. van der Merwe, eds. (Kluwer Academic Publishers, Dordrecht, 1997) p. 77.

22. This is the essence of the quantum non-cloning theorem, see W.K. Wootters and W.H. Zurek, A single quantum cannot be cloned, Nature 299, 802 (1982); D. Dieks, Communication by EPR devices, Phys. Lett. 92A, 271 (1982).

23. D.M. Greenberger, M.A. Horne, and A. Zeilinger, Going beyond Bell's theorem, in Bell's Theorem, Quantum Theory, and Conceptions of the Universe, M. Kafatos, ed., (Kluwer, Dordrecht, 1989) p. 69; N.D. Mermin, Quantum mysteries revisited, Am. J. Phys. 58, 731 (1990).

24. D.P. DiVincenzo, Quantum computation, Science 270, 255 (1995); I.L. Chuang, R. Laflamme, P.W. Shor, and W.H. Zurek, Quantum computers, factoring, and decoherence, ibid., p. 1633; C.H.Bennett, Quantum information and computation, Phys. Today 48, 24 (October, 1995).

25. N.J. Cerf and C. Adami, Entropic Bell inequalities, Phys. Rev. A 55, 3371 (1997). 